\title{
State-selected ion-molecule reactions with Coulomb-crystallized molecular ions in traps
}

\author{
Xin Tong, Tibor Nagy, Juvenal Yosa Reyes, Matthias Germann, Markus Meuwly*, Stefan Willitsch* \\ Department of Chemistry, University of Basel, Klingelbergstrasse 80, 4056 Basel, Switzerland
}

\begin{abstract}
त

State-selected Coulomb-crystallized molecular ions were employed for the first time in ion-molecule reaction studies using the prototypical charge-transfer process $\mathrm{N}_{2}^{+}+\mathrm{N}_{2} \rightarrow \mathrm{N}_{2}+\mathrm{N}_{2}^{+}$as an example. By preparing the reactant ions in a well-defined rovibrational state and localizing them in space by sympathetic cooling to millikelvin temperatures in an ion trap, state- and energy-controlled reaction experiments with sensitivities on the level of single ions were performed. The experimental results were interpreted with quasi-classical trajectory simulations on a six-dimensional potential-energy surface which provided detailed insight into translation-to-rotation energy transfer occurring during charge transfer between $\mathrm{N}_{2}$ and $\mathrm{N}_{2}^{+}$.

त
\end{abstract}

Keywords:

State-selected ion-molecule reactions, Coulomb crystals, cold molecules, reactive surfaces, charge transfer, adiabatic reactive molecular dynamics, translation-to-rotation energy transfer

\section{Introduction}

Achieving full control over the internal quantum states as well as the collision energy of the reaction partners to probe the detailed state-to-state dynamics and energy dependence of chemical processes has been one of the longstanding aspirations in gas-phase chemical studies. In the past, a range of experimental methods has been developed to address these objectives, e.g., internal cooling of the reactants in supersonic expansions, experiments with crossed molecular beams at variable relative velocities and laser preparation of the reactants in specific quantum states (see, e.g., Refs. 1, 2, 3] and literature cited therein). Very recently, new techniques have been established which allow the preparation of molecules at extremely low translational temperatures $<1 \mathrm{~K}$ and at the same time enable an unprecedented degree of control over their kinetic energy [4, 5, 6. In conjunction with the simultaneous preparation of the molecules in well-defined rotational-vibrational and even hyperfine states, "cold molecules" methods are now starting to pave the way for studies of collisional processes and chemical reactions in new physical regimes and at levels of detail and sensitivity which have not been possible before [7, 8, 9, 10, 11].

Whereas most efforts have so far concentrated on neutral molecules, techniques for cooling and controlling molecular ions have recently made impressive progress as well. From an experimental perspective, the sensitivity of the

\footnotetext{
*Corresponding authors. E-mail: m.meuwly@unibas.ch, stefan.willitsch@unibas.ch
}

ion motion to weak stray electric fields in the apparatus renders the precise control of their kinetic energy difficult. This problem can be overcome by trapping the ions and cooling them sympathetically to millikelvin temperatures by the interaction with co-trapped laser-cooled atomic ions [12. Under these conditions, the ions localize in space to form ordered structures usually referred to as "Coulomb crystals" in which it is possible to observe, manipulate and address single ions [6, 13].

Moreover, the preparation of molecular ions in welldefined quantum states is experimentally challenging and indeed only a handful of reaction studies with rotationally state-selected ions have been reported thus far (see, e.g., [14, 15, 16, 17, 18]). Only very recently it has become possible to prepare Coulomb-crystallized molecular ions in well-defined internal quantum states [19, 20, 21, 22, so that a simultaneous control over the kinetic energies, positions and internal states of the ions can now be achieved.

In the present article, we report the first study of a chemical reaction with rovibrationally state-selected Coulomb-crystallized molecular ions. We investigate the prototypical symmetric charge-transfer (CT) reaction $\mathrm{N}_{2}^{+}+\mathrm{N}_{2} \rightarrow \mathrm{N}_{2}+\mathrm{N}_{2}^{+}$between state-prepared sympathetically-cooled $\mathrm{N}_{2}^{+}$ions and internally cold $\mathrm{N}_{2}$ molecules from a supersonic expansion at a well-defined collision energy.

The mechanism and kinetics of this reaction have been studied previously, see, e.g., Refs. [23, 24, 25, 26, 27, 28] and references therein. At low energies, the reaction was found to proceed via a long-lived $\mathrm{N}_{4}^{+}$reaction complex which forms at the collision (Langevin) rate 24, 


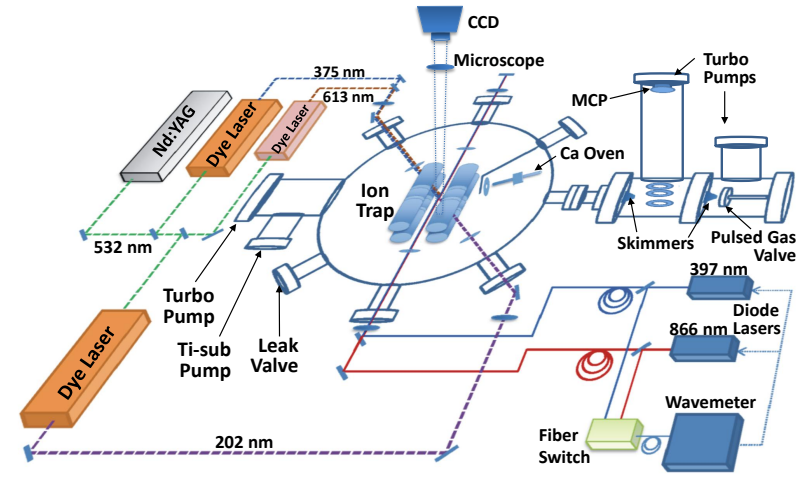

Figure 1: Schematic of the experimental setup.

25. 28. Using isotopically labeled nitrogen ions, Frost et al. showed that near-thermal collisions between $\mathrm{N}_{2}^{+}$ ions and $\mathrm{N}_{2}$ molecules in their vibrational ground states lead to a symmetric $\mathrm{CT}$ with a rate constant of $k=$ $4.2 \times 10^{-10} \mathrm{~cm}^{3} \mathrm{~s}^{-1}$ which amounts to one half of the collision rate constant 25. This observation was rationalized in terms of a symmetric sharing of the charge between the two $\mathrm{N}_{2}$ moieties in the $\mathrm{N}_{4}^{+}$reaction complex so that the probability for CT amounts to $50 \%$ upon its breakup. Vibration-to-vibration (V-V) and vibration-to-translation (V-T) energy transfer occurring during CT have been investigated in this system both experimentally and theoretically, see Refs. [25, 26, 27, 29, 30] and references therein. Kato et al. observed multi-quantum vibrationalenergy transfer at near-thermal collision energies which was rationalized in terms of energy redistribution within the strongly bound $\mathrm{N}_{4}^{+}$reaction complex [26]. Also, evidence for vibration-to-rotation and translation $(\mathrm{V}-\mathrm{R}, \mathrm{T})$ energy transfer was found.

To our knowledge, the present work is the first to achieve rotational state selection of the reactant ions and rotational resolution in the analysis of the product-ion state populations allowing to gain insight into the dynamics of translation-to-rotation (T-R) energy transfer occurring during CT. The Coulomb-crystal technology employed in the present study enabled us to monitor reactive collisions in small ensembles of 20 to 30 state-selected, spatially localized $\mathrm{N}_{2}^{+}$ions with single-particle sensitivity. The experimental results were interpreted with quasi-classical trajectory calculations on a fully-dimensional potential-energy surface (PES) which provided insight into the charge- and energy-transfer dynamics underlying this fundamental reaction.

\section{Experimental methods}

The experimental setup for generating quantum-state selected Coulomb-crystallized $\mathrm{N}_{2}^{+}$ions (see Fig. 1) has been described in detail in previous publications [19. 20. Briefly, $\mathrm{N}_{2}^{+}$ions in the rovibronic ground state
$X^{+}{ }^{2} \Sigma_{g}^{+}, v^{+}=0, N^{+}=0, F_{1}$ were prepared by a $\left[2+1^{\prime}\right]$ resonance-enhanced threshold-photoionization sequence via the $a^{\prime \prime}{ }^{1} \Sigma_{g}^{+}, v^{\prime}=0, J^{\prime}=2$ intermediate level of neutral $\mathrm{N}_{2}$ as shown in Fig. 2 (a). Here, $v^{+}$and $v^{\prime}$ $\left(N^{+}\right.$and $\left.J^{\prime}\right)$ stand for the vibrational (rotational) quantum numbers of the cationic ground and neutral intermediate states, respectively, and $F_{1}$ denotes the spin-rotational component. The $\mathrm{N}_{2}^{+}$ions were generated inside a linear quadrupole ion trap 6, 13] from a collimated, pulsed supersonic molecular beam of pure $\mathrm{N}_{2}$ gas. The molecular beam was positioned at a distance of $\approx 400 \mu \mathrm{m}$ from the Coulomb crystal in order to prevent collisions between ions and neutrals during the loading phase. The rotational temperature of the $\mathrm{N}_{2}$ molecules in the beam was determined to be $T_{\text {rot }} \approx 10 \mathrm{~K}$ by resonance-enhanced multiphoton-ionization (REMPI) spectroscopy [20] corresponding to populations of $50,25,22$, and $3 \%$ in the $J=0,1,2,3$ rotational states. The beam velocity was estimated to be $\approx 787 \mathrm{~ms}^{-1}$ from flow-dynamics models 31 .

Immediately after their generation, typically 25 stateselected $\mathrm{N}_{2}^{+}$ions were sympathetically cooled to translational temperatures of $\approx 10 \mathrm{mK}$ by the interaction with laser-cooled $\mathrm{Ca}^{+}$ions to form bi-component Coulomb crystals [6, 13]. $\mathrm{Ca}^{+}$ions were produced in the center of the trap by non-resonant photoionization of Ca atoms emanating from a Ca oven. The $\mathrm{Ca}^{+}$ions were laser cooled on the $4 s^{2} S_{1 / 2} \rightarrow 4 p{ }^{2} P_{1 / 2}$ transition using diode-laser radiation at $397 \mathrm{~nm}$. Another diode-laser beam at $866 \mathrm{~nm}$ was used to repump population on the $3 d^{2} D_{3 / 2} \rightarrow 4 p^{2} P_{1 / 2}$ transition to close the laser cooling cycle. The resulting $\mathrm{Ca}^{+} / \mathrm{N}_{2}^{+}$bi-component crystals were imaged by collecting the spatially resolved laser-cooling fluorescence of the $\mathrm{Ca}^{+}$ ions using a microscope coupled to camera. In the images, the positions of the non-fluorescing molecular ions are visible as a dark region in the center of the crystals, see Fig. 3 (a).

Reactive collisions between state-selected $\mathrm{N}_{2}^{+}$ions and neutral $\mathrm{N}_{2}$ molecules were initiated by overlapping the molecular beam with the bi-component Coulomb crystal immediately after ion loading and sympathetic cooling. The collision energy $E_{\text {col }} \approx 0.045 \mathrm{eV}$ in the present experiments was entirely dominated by the kinetic energy of the $\mathrm{N}_{2}$ molecules in the beam. Neglecting the small initial rotational excitation of neutral $\mathrm{N}_{2}$, the maximum kinetic energy of the product ions cannot exceed the total kinetic energy of the reactants which is much smaller than the trap depth $(>2 \mathrm{eV})$. Consequently, the product ions remained trapped and were sympathetically re-cooled into the Coulomb crystal. Because the product ions were chemically identical to the reactant ions, their presence manifested itself in a time-dependent increase of the population in rotationally excited states in the ensemble of sympathetically-cooled $\mathrm{N}_{2}^{+}$ions. Vibrational excitation of the products was precluded on energetic grounds. Rate constants were determined by measuring the $\mathrm{N}_{2}^{+}$spinrotational state populations as a function of the effective 


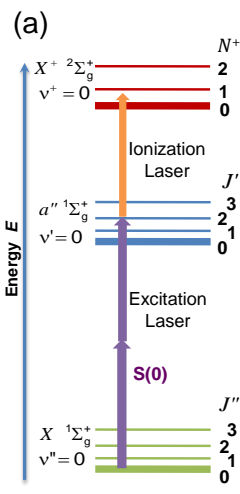

(b)

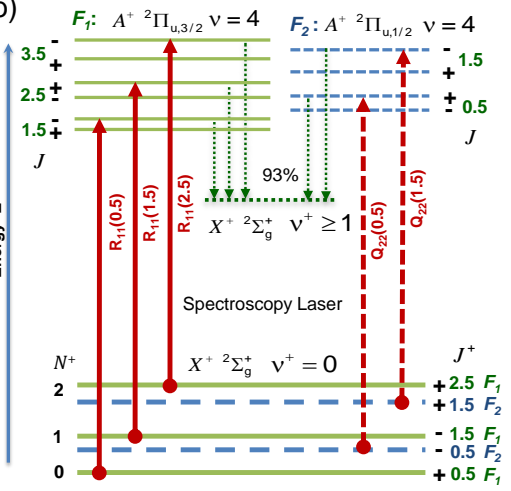

Figure 2: (a) Resonance-enhanced threshold-photoionization scheme used to generate $\mathrm{N}_{2}^{+}$ions in the $X^{+}{ }^{2} \Sigma_{g}^{+}, v^{+}=0, N^{+}=0, F_{1}$ spin-rovibrational ground state. (b) Laser-induced charge-transfer (LICT) scheme for probing the populations in the spin-rotational levels $N^{+}, F_{1,2}$ of the vibronic ground state of $\mathrm{N}_{2}^{+}$produced by CT reactions. See text for details.

time of reaction with $\mathrm{N}_{2}$ molecules from the beam and fitting the results to a kinetic model as detailed in Sec. 4.

The rotational-state populations of the $\mathrm{N}_{2}^{+}$product ions were probed by optical pumping to vibrationally excited levels to promote CT reactions with Ar atoms which are energetically forbidden in the vibrational ground state (laser-induced charge-transfer (LICT) spectroscopy [19, 20]). LICT was initiated by exciting transitions to selected rotational levels of the $X^{+}{ }^{2} \Sigma_{g}^{+}, v^{+}=0 \rightarrow$ $A^{+}{ }^{2} \Pi_{u}, v=4$ state with a pulsed dye laser operating at $613 \mathrm{~nm}$, see Fig. 2 (b). Subsequent fluorescent decay lead to the population of vibrationally excited states $v^{+} \geq 1$ in the electronic ground state with a probability of $93 \%$. The removal of vibrationally excited $\mathrm{N}_{2}^{+}$ions by CT with Ar gas leaked into the chamber was directly observed by the reduction of the core of non-fluorescing ions in the images, see Fig. 3 (b). The number of $\mathrm{N}_{2}^{+}$ions lost by LICT represented a direct measure of the population in the initially excited spin-rotational state. This number was determined by a comparison of the experimental fluorescence images with simulated images generated from molecular-dynamics simulations [19, 20], see Fig. 3 (a).

\section{Theoretical methods}

\subsection{Potential energy surfaces}

The ground state six-dimensional potential-energy surface (PES) of the $\mathrm{N}_{2}-\mathrm{N}_{2}^{+}$system was computed at the UCCSD level 32, 33] with Dunning's correlation consistent polarized valence basis set (cc-pVTZ) 34 using Gaussian03 35. Single-point calculations were performed for 5565 non-equivalent geometries with energies up to $1.9 \mathrm{eV}$ relative to the minimum of the $\mathrm{N}_{4}^{+}$complex on a nonequidistant rectangular grid including the N-N separations $r_{1}$ and $r_{2}$ in $\mathrm{N}_{2}$ and $\mathrm{N}_{2}^{+}$, respectively, the center of mass distance $R$ between the two diatomic molecules, and the angles $\theta_{1}, \theta_{2}$ and $\phi$ (see the inset in Fig. 6). The calculated well-depth $\left(D_{\mathrm{e}}\right)$ of the complex is $1.254 \mathrm{eV}$, in good agreement with previous even higher-level theoretical estimates $(1.26 \mathrm{eV}$ at the $(\mathrm{RCCSD}(\mathrm{T}) / \mathrm{vqz}(\mathrm{spdfg})$ level of theory [36]). For comparison, the experimentally determined dissociation energy of $\mathrm{N}_{4}^{+}$is $D_{0}=1.06 \mathrm{eV}$ [37.

The global PES was represented by three surfaces, referred to as one "bound" $\left(\mathrm{N}_{4}^{+}\right)$and two "unbound" ones $\left(\mathrm{N}_{2}-\mathrm{N}_{2}^{+}\right.$and $\left.\mathrm{N}_{2}^{+}-\mathrm{N}_{2}\right)$. They were distinguished by the distance $R$ and the localisation of the majority of the charge. For $R \leq 7.09 a_{0}(3.75 \AA)$ the system is considered to be bound whereas for $R \geq 7.56 a_{0}(4.0 \AA)$ it is unbound. The intermediate region $\left(7.09-7.56 a_{0}\right)$ represents the transition region during the dynamics, where the surfaces are connected by a smooth switching-function 38 .

To allow a) bond formation between any two atoms of $\mathrm{N}_{2}$ and $\mathrm{N}_{2}^{+}$and b) dissociation of the complex into either the charge-preserving or charge-transferring state at least 8 force fields (FFs) are needed for the bound state and 4 FFs are necessary for each of the unbound states. These FFs are related to each other through permutation of their parameters. Within each set of FFs always the lowest energy surface is followed, except for when they are close in energy (within $\approx 0.02 \mathrm{eV}$ ) in which case they are smoothly joined by an energy difference-based switching function 38 .

Morse and Lennard-Jones potentials were used for the bond stretches and the intermolecular interactions, respectively, for the unbound states. Morse parameters for the isolated molecules were determined from PES scans at the UCCSD level of theory. For the bound state, Morse potentials were used for all bonds of the complex and LennardJones and electrostatic potentials between the edge atoms.

The force field parameters were fitted to the $5565 \mathrm{ab}$ initio points with a simplex algorithm 39. The ab initio energies for the unbound region were reproduced with a root-mean-square-deviation $(\mathrm{RMSD})$ of $\approx 0.013 \mathrm{eV}$. It was considerably more difficult to obtain a good fit for the bound state due to strong angular dependence of the potential. The final fit had an RMSD of $\approx 0.06 \mathrm{eV}$ by using two minimal symmetrized sets of FFs (twice 8, altogether 16 FFs). Figure 6 shows an example of the quality of the fit. The well depth of the bound state was exactly reproduced by the global surface. In the following, the bound state FFs are numbered 1-16, whereas those for the unbound states are labeled 17-20 and 21-24, respectively.

\subsection{Molecular-dynamics (MD) simulations}

For the quasi-classical trajectory calculations a code with provisions for adiabatic reactive MD (ARMD) 40] was used. The Hamiltonian equations of motion were solved in Cartesian coordinates using the adaptive timestep Modified Extended Backward Differentiation Formulas method (MEBDFSO) 41].

The dynamics was initiated in the unbound state (FFs 2124). Between centre-of-mass separations of $R=7.56 a_{0}$ 
and $R=7.09 a_{0}$ the momentarily active unbound surface was smoothly switched to the bound FFs (numbers 1-16). The complex is considered to be formed for $R \leq 7.09 a_{0}$ and the dynamics is continued in the bound state. When a separation of $R=7.56 a_{0}$ is reached again, it is determined which unbound $\mathrm{FF}$ is lowest in energy at the corresponding geometry and the dynamics is followed on this FF by smoothly switching from the bound state. Following the dynamics further, the system either can return to the bound state (recrossing) or it can decay into either $\mathrm{N}_{2}+\mathrm{N}_{2}^{+}$(charge preserving, FFs 21 to 24 ) or $\mathrm{N}_{2}^{+}+\mathrm{N}_{2}$ (charge transferring, FFs 17-20). The dynamics is followed until either the lifetime of the bound state exceeded $100 \mathrm{ps}$ or the initial separation of the two fragments was reached again. Once the complex survives a few vibrational periods, energy is expected to have randomized completely. Our results show that if the complex survives for at least $0.4 \mathrm{ps}$ the correlation between the reactant and product states is negligible.

\subsection{Analysis of final sates}

As a consequence of using classical dynamics, products are formed with rovibrational energies and angular momenta which correspond to fractional quantum numbers. Also, products having less than zero-point vibrational energy (ZPE) can be formed. This ZPE leakage is a shortcoming of quasi-classical simulations and various methods were proposed for either avoiding or correcting it [42, 43, 44]. In the present study, only trajectories which satisfy certain constraints in the product states, i.e., the total ZPE of the two molecules should be conserved within $\pm 10 \%$, were further analyzed.

\section{Results and Discussion}

\subsection{Product rotational-state distributions}

Fig. 3 (b) shows LICT measurements probing the populations in the $N^{+}=0, F_{1}$ and $N^{+}=1, F_{1}$ spin-rotational states as a function of the effective time of reaction with neutral $\mathrm{N}_{2}$ molecules from the molecular beam. For the $N^{+}=0$ state, a marked decrease of the LICT efficiency can be observed with increasing reaction time, indicating the removal of the initially prepared $\mathrm{N}_{2}^{+}, N^{+}=0$ ions by $\mathrm{CT}$ with $\mathrm{N}_{2}$. Conversely, an increase in the LICT efficiency out of the $N^{+}=1$ state can be observed, indicating the generation of ions in rotationally excited states as a consequence of the reactive collisions.

LICT measurements on the population of the $N^{+}=$ $0,1,2 F_{1,2}$ levels as a function of the reaction time are shown in Fig. 4. Two independent LICT measurements were performed for each spin-rotational level at four different effective reaction times. As a general trend, a decrease of the population in $\mathrm{N}^{+}=0$ in favor of an increase of the population in $N^{+} \geq 1$ was observed. This result indicates that product ions over the whole range of rotationally excited states probed in the present study were generated (a)

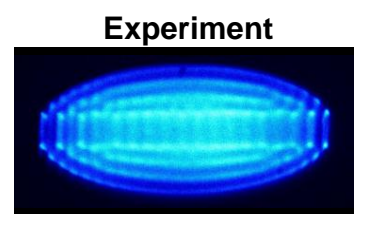

(b) $N^{+}=0, F_{1}$

$t$ (s)

0

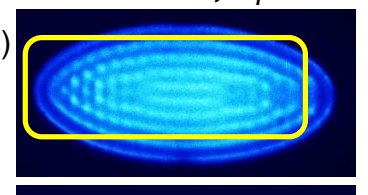

1.8

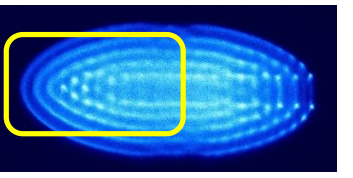

3.6

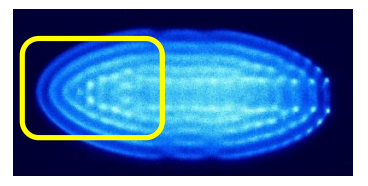

5.4

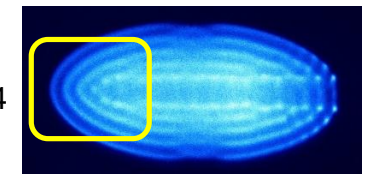

Simulation

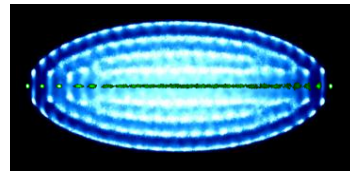

$N^{+}=1, F_{1}$
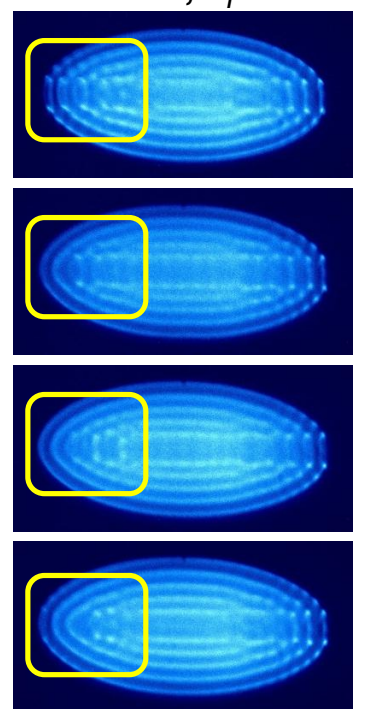

Figure 3: (a) Experimental false-color fluorescence image and molecular-dynamics simulation of a $\mathrm{Ca}^{+} / \mathrm{N}_{2}^{+}$bicomponent Coulomb crystal before reaction. The spatial distribution of the nonfluorescing molecular ions has been made visible in green in the simulated image. (b) LICT experiments probing the populations in the $\mathrm{N}_{2}^{+} N^{+}=0, F_{1}$ and $N^{+}=1, F_{1}$ spin-rotational states. The panels show fluorescence images obtained after LICT as a function of the effective time of reaction $t$ with neutral $\mathrm{N}_{2}$ molecules from the molecular beam. The LICT efficiency (corresponding to the number of non-fluorescing ions removed from the crystal in the boxed areas) decreases in $N^{+}=0$ and increases in $N^{+}=1$ with increasing reaction time, indicating the generation of rotationally excited $\mathrm{N}_{2}^{+}$ions from the $\mathrm{CT}$ reaction $\mathrm{N}_{2}^{+}+\mathrm{N}_{2}$. See text for details.

as a consequence of CT between $\mathrm{N}_{2}^{+}+\mathrm{N}_{2}$. Because the neutral products leave the trap, no conclusions on their rotational-state distribution can be drawn.

The observed redistribution of population in the ensemble of Coulomb-crystallized $\mathrm{N}_{2}^{+}$ions is the result of a sequence of $\mathrm{CT}$ reactions with neutral molecules from the beam. The initially state-selected $\mathrm{N}_{2}^{+}$ions undergo $\mathrm{CT}$ collisions with rotationally cold $\mathrm{N}_{2}$ molecules. The resulting $\mathrm{N}_{2}^{+}$product ions are sympathetically cooled into the Coulomb crystal whereupon they in turn can undergo collisions. In this way, the ensemble of originally state-selected $\mathrm{N}_{2}^{+}$ions in the Coulomb crystal is lost and replaced with rotationally excited product ions.

Close inspection of the data in Fig. 4 reveals two important details. First, already in the beginning of the experiment when most of the reactive collisions occur with ions in $N^{+}=0$, the generation of ions in all rotational levels probed in the present study can be observed. This 


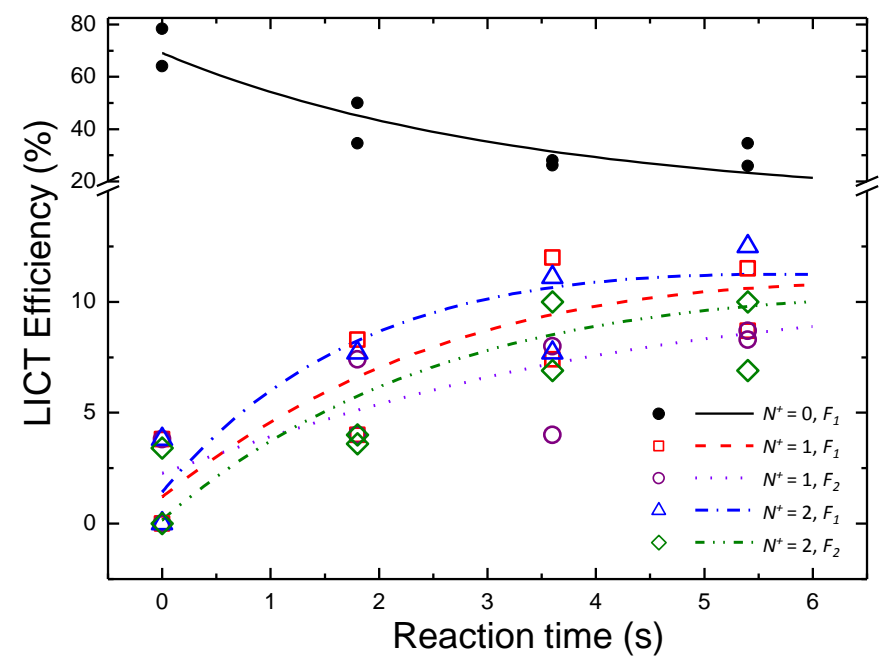

Figure 4: LICT efficiencies reflecting the populations in the lowest five spin-rotational levels of $\mathrm{N}_{2}^{+}$as a function of the reaction time with neutral $\mathrm{N}_{2}$ molecules. Over the course of the reaction, the initial ensemble of state-selected $\mathrm{N}_{2}^{+}$ions is replaced by rotationally excited product ions. The lines are a fit of the data to the kinetic model shown in Fig. 5 (a).

observation suggests that the production of product ions is feasible over a broad range of rotational states by reactions with $\mathrm{N}_{2}^{+}$in $N^{+}=0$. The relevant rates appear to be of a similar magnitude, at least over the range of product states probed in the present study. Second, for a specific rotational level $N^{+}$, there appears to be a slight preference for the generation of the $F_{1}$ spin-rotational component in comparison to the $F_{2}$ component.

\subsection{Kinetics}

The data were analyzed in terms of kinetic models taking into account CT collisions of the initially prepared $\mathrm{N}_{2}^{+}\left(N^{+}=0\right)$ reactant ions as well as consecutive reactions of the product $\mathrm{N}_{2}^{+}$ions, see Fig. 5. Because the number density of neutral $\mathrm{N}_{2}$ in the molecular beam is much larger than the number density of the $\mathrm{N}_{2}^{+}$ions in the trap, pseudo-first-order kinetics was assumed in both models.

Based on the observation that all product rotational states probed in the present study $\left(N^{+} \leq 2\right)$ seem to be produced with similar rates, it was assumed in a first model that all processes of the form $\mathrm{N}_{2}^{+}\left(N^{+}\right)+\mathrm{N}_{2}(J) \rightarrow \mathrm{N}_{2}(\bar{J})+\mathrm{N}_{2}^{+}\left(\bar{N}^{+}\right)$(bars indicate the rotational quantum numbers after the decay of the reaction complex) occur with the same $k$, see Fig. 5 (a). The complete loss of population from the manifold of states with $N^{+} \leq 2$ was taken into account by an effective loss rate constant $k^{\prime}$. In the absence of detailed information on the neutral product state distribution, $k$ and $k^{\prime}$ represent effective rate constants averaged over all neutral product states. The spin-rotational level populations $n_{i}$ were ob-

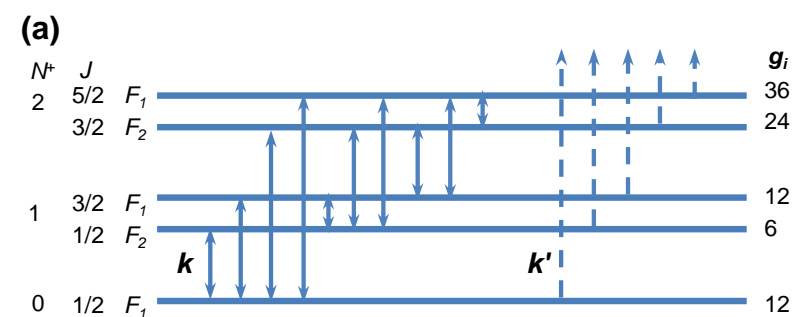

(b)

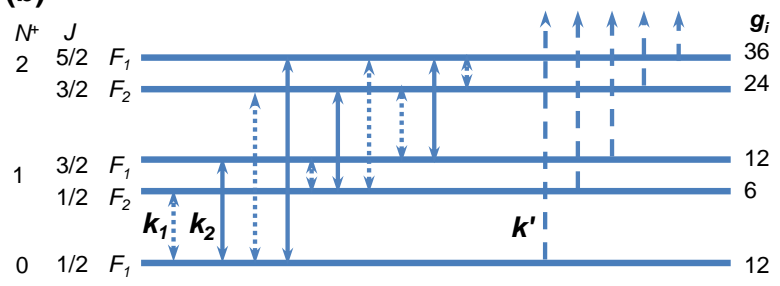

Figure 5: Schematic of kinetic models used to fit the redistribution of rotational populations in the ensemble of sympathetically-cooled $\mathrm{N}_{2}^{+}$ions as a consequence of CT collisions. Model assuming (a) an uniform rate constant $k$ and (b) different rate constants $k_{1}$ and $k_{2}$ for processes connecting unlike and like spin-rotational levels $F_{1,2}$ in the reactant and product ions, respectively. See text for details. $g_{i}$ denotes the degeneracies of the spin-rotational levels including nuclear-spin statistical weights.

tained from the set of rate equations

$$
\frac{\mathrm{d} n_{i}}{\mathrm{~d} t}=k \sum_{j \neq i}\left(g_{i} n_{j}-g_{j} n_{i}\right)-k^{\prime} n_{i}
$$

where $g_{i, j}$ represent degeneracy factors (see Fig. 5). The initial state populations $n_{i}(t=0)$ were also treated as a fit parameter, accounting for the uncertainty in determining the starting time of the measurement following the realignment of the molecular beam after ion loading (see Sec. 2). The fit of this model to the experimental data yielded the pseudo-first-order rate constants $k=0.0023(4) \mathrm{s}^{-1}$ and $k^{\prime}=0.04(3) \mathrm{s}^{-1}$. Because the density of neutral molecules in the molecular beam is not precisely known, it was not possible to obtain second-order rate constants from these results. Previous rate measurements with thermal samples of $\mathrm{N}_{2}$ gas leaked into the chamber [20] were consistent with the result obtained by Frost et al. [25] that the total CT rate amounts to one half of the Langevin collision rate $k_{L}=8.3 \times 10^{-10} \mathrm{~cm}^{3} \mathrm{~s}^{-1}$.

The time-dependent populations of the $N^{+}=$ $0,1,2, F_{1,2}$ states computed with the kinetic model using the fitted parameters are shown in Fig. 4. The agreement between calculated and experimental level populations is satisfactory given the spread of the measured level populations. The agreement vindicates the assumption that the state-specific rate constants do not (or only weakly) depend on the rotational state of the product ion within the uncertainty limits of the present measurement and the range of states studied. The kinetic model also reproduces the preferential generation of ions in the $F_{1}$ compared to the $F_{2}$ spin-rotational components of the same $N^{+}$state 


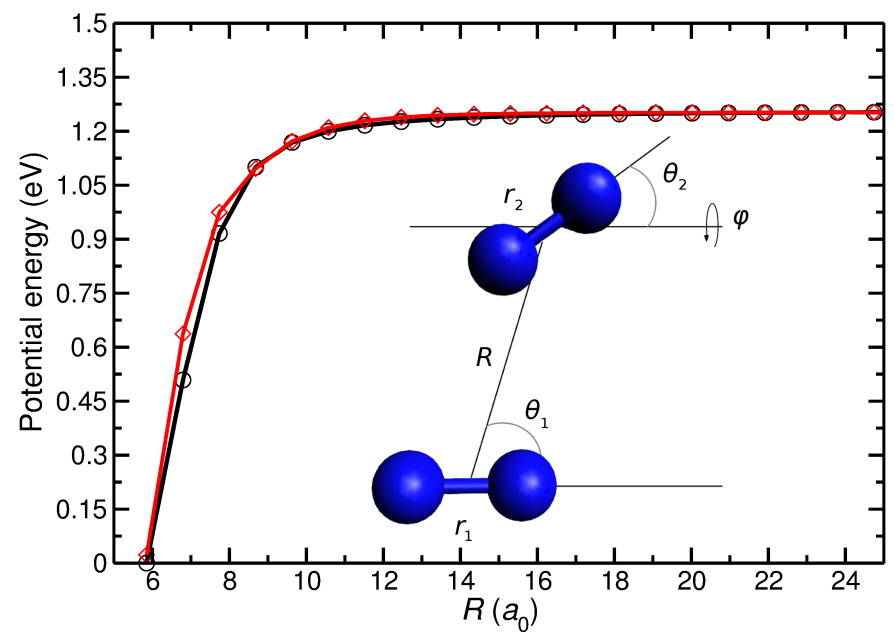

Figure 6: Comparison of the UCCSD/cc-pVTZ (black) and fitted energies (red) for linear structures with $r_{1}=r_{2}=2.08 a_{0}$ as a function of $R$. The inset shows the relevant coordinates scanned in the ab initio calculations.

suggesting that this effect is caused by the higher statistical weight of the $F_{1}$ components.

To check the validity of this model, its main simplification, i.e., the restriction to a "universal" state-to-state rate constant $k$, was relaxed. In a second kinetic model (see Fig. 5(b)), different rate constants $k_{1}$ and $k_{2}$ for reactions connecting states with different and like spin-rotational labels $F_{1,2}$, respectively, were assumed to account for the preferential production of product ions in the $F_{1}$ levels. The fit yielded rate coefficients $k_{1}=0.0023(9) \mathrm{s}^{-1}, k_{2}=$ $0.0022(7) \mathrm{s}^{-1}$ and $k^{\prime}=0.04(3) \mathrm{s}^{-1}$. The results obtained for $k_{1}$ and $k_{2}$ agree with each other and with the value of $k$ within the uncertainty limits, supporting the conclusion that the increased production rates of ions in the $F_{1}$ levels is indeed solely caused by the higher statistical weights associated with these channels.

\subsection{Trajectory calculations}

Quasi-classical trajectory calculations were performed to interpret the experimental findings and gain further insight into the energy-transfer mechanisms involved in the reaction. Initial states for the trajectory calculations correspond to semi-classically quantized states of rotating Morse oscillators [45. In the experiment the molecules are exclusively in their ground vibrational state $(v=0)$. The initial rotational angular momentum $N^{+}$of $\mathrm{N}_{2}^{+}$ions was set to zero as prepared in the experiment whereas that of $\mathrm{N}_{2}$ was randomly sampled according to the experimentally measured distribution (see Sect. 2). Initial coordinates and momenta were generated by randomly sampling the phase-space distribution of the rotating Morse-oscillators and the spatial orientation of each molecule was taken from a uniform distribution within $4 \pi$ steradian. The magnitude of the angular velocity for a given vibrational phase was set to the angular momentum calculated from

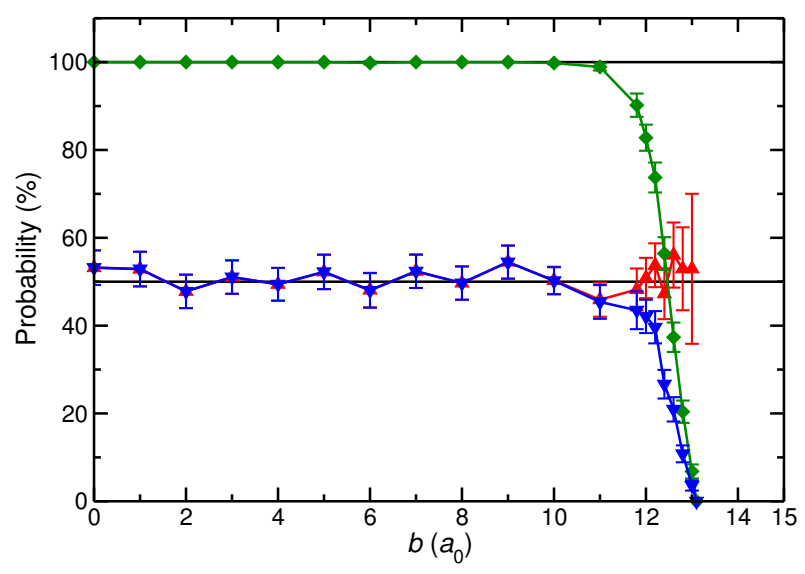

Figure 7: Opacity functions for complex formation (green diamonds) and charge transfer (blue triangles), and probability curve for charge transfer (red triangles) if the complex is formed. Error bars correspond to a $2 \sigma$ standard deviation.

$L=(J(J+1))^{1 / 2} \hbar$ and its direction was randomly sampled from a uniform distribution in the plane perpendicular to the axis of the molecule.

Impact parameters $b$ were between 0 and $15 a_{0}$ in steps of $1 a_{0}$, and in steps of $0.2 a_{0}$ between 12 and $13 a_{0}$ where the opacity function drops to zero (see below). The initial separation of the center of mass of the two molecules was $20 \AA\left(\approx 38 a_{0}\right)$, for which the intermolecular interaction energy was less than $1.4 \times 10^{-4} \mathrm{eV}$. According to the Langevin-Gioumousis-Stevenson (LGS) model of ionmolecule reactions the rate does not depend on the relative velocity of the partners [46]. Therefore, the relative velocity of the colliding molecules was set to the calculated beam flow rate $(787 \mathrm{~m} / \mathrm{s})$ without dispersion.

To validate the simulations, the probability of complex formation and the probability of CT were determined for a range of impact parameters $b$ based on 500-1000 trajectories at each value (see Figure 7). According to our quasi-classical model, the probability for CT was found to be $50 \%$ (within the statistical uncertainty) up to impact parameters $b=9 a_{0}$. The CT probability starts dropping slowly between 10 and $12 a_{0}$, and above $12 a_{0}$ it decays steeply to zero around $13 a_{0}$. The probability of complex formation is $100 \%$ up to $9 a_{0}$ and drops proportionally to the CT probability. Once the complex is formed, the probability for $\mathrm{CT}$ is $\approx 50 \%$ regardless of the impact parameter. This finding is expected based on symmetry arguments and considering that rapid charge-redistribution takes place before the complex decays. It is also in agreement with previous experimental results [25].

From the integrated opacity function an integral cross section for CT of $\sigma_{\text {tot }}=(243 \pm 19) a_{0}^{2}$ was obtained. Multiplication with the relative velocity $\left(787 \mathrm{~m} \mathrm{~s}^{-1}\right)$ yields $k=(5.36 \pm 0.42) \times 10^{-10} \mathrm{~cm}^{3} \mathrm{~s}^{-1}$ for the second order rate coefficient for CT. This value compares favorably with previous experimental results of $4.24 \times 10^{-10} \mathrm{~cm}^{3} \mathrm{~s}^{-1}$, $6.6 \times 10^{-10} \mathrm{~cm}^{3} \mathrm{~s}^{-1}$ and $5.0 \times 10^{-10} \mathrm{~cm}^{3} \mathrm{~s}^{-1}$ obtained 

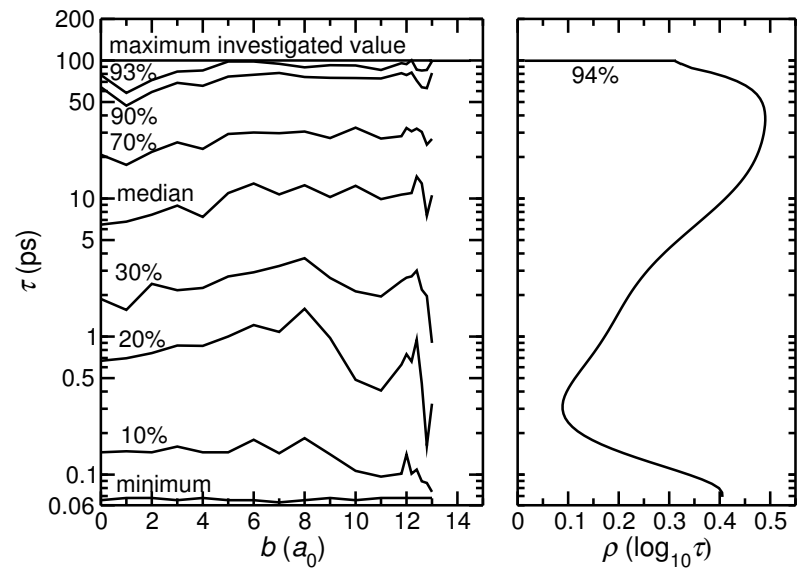

Figure 8: Percentiles representing the distribution of lifetimes $\tau$ for the $\mathrm{N}_{4}^{+}$complex as a function of the impact parameter $b$ (left panel) and integrated probability density $\rho$ (right panel). The median lifetime is around $10 \mathrm{ps}$, the complex decays within $100 \mathrm{ps}$ in $\approx 94 \%$ of all observed cases.

for the CT reaction between ${ }^{15} \mathrm{~N}_{2}^{+}(v=0)$ and ${ }^{14} \mathrm{~N}_{2}(v=0)$ [25, 47, 48].

The lifetime of the complex was determined as the time difference between the last and the first crossing of the surface separation radius $7.09 a_{0}$ (see Sect. 3.1. As Figure 8 suggests, the median lifetime of $\mathrm{N}_{4}^{+}$is $\approx 10 \mathrm{ps}$ and does not or only slightly depends on $b$. Lifetimes up to $100 \mathrm{ps}$ are found for $94 \%$ of the trajectories which qualitatively agree with previous estimates [24, 49].

\subsection{Translation-to-rotation energy transfer}

The salient quantity which can be extracted from the trajectories to aid in the interpretation of the experimental results is the distribution of rotational angular momentum $\rho\left(\bar{N}^{+}\right)$of the $\mathrm{N}_{2}^{+}$products after complex formation and decay. Figure 9 reports $\rho\left(\bar{N}^{+}\right)$and suggests that for the majority $(97 \%)$ of cases $\bar{N}^{+} \leq 13$. On energetic grounds, a total of $0.045 \mathrm{eV}$ is available in collisional energy for the reactants, and the initial rotational quantum number of $\mathrm{N}_{2}$ can be as high as 3 (corresponding to a rotational energy of $\approx 0.003 \mathrm{eV}$ ), which implies $\bar{N}^{+} \leq 14$. The $\mathrm{N}_{2}^{+}$ products are predominantly formed in excited rotational states, which supports the experimental findings of depletion of the $\mathrm{N}_{2}^{+}$ground state population caused by reactive collisions with the molecules in the beam. Note that the theoretical product state distribution cannot be compared directly with the experimental findings reported in Fig. 4. because the $\mathrm{N}_{2}^{+}$ions probed in the experiment result from a sequence of $\mathrm{CT}$ reactions, whereas the simulations only reflect single collision events.

However, the trajectory calculations clearly reproduce the rotational excitation of the products observed in the experiment and reveal the mechanisms of the underlying T-R energy transfer occurring during CT. The reactant molecules approach each other in a random orientation
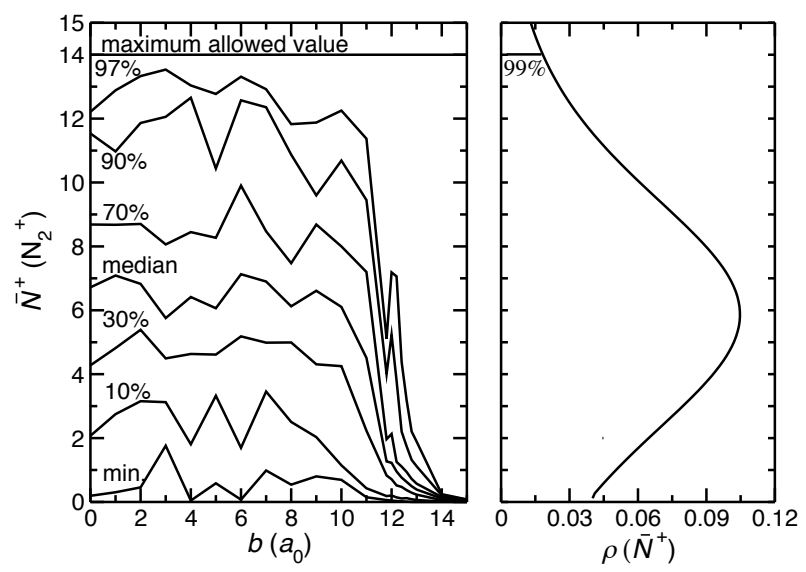

Figure 9: Percentiles representing the rotational-state distributions for the $\mathrm{N}_{2}^{+}$products after breakup of the reaction complex as a function of the impact parameter $b$ (left panel) and integrated probability density $\rho\left(\bar{N}^{+}\right)$(right panel). The products are predominantly formed in rotationally excited states.

and are accelerated towards the potential well of the linear reaction complex. The torque exerted while forcing the reactants towards a linear configuration excites a counterrotation of the $\mathrm{N}_{2}$ moieties which results in highly excited bending and torsional vibrations of the complex. Moreover, anharmonic couplings lead to a practically complete redistribution of the available energy over all vibrational degrees of freedom during the lifetime of the complex. Upon its breakup, large-amplitude bending and torsional vibrations are converted into product rotations. Representative trajectories, projected on the PES, are shown in Fig. 10 to illustrate this effect.

Close inspection of the trajectories reveals the likely presence of additional processes resulting in the rotational excitation of the products, including rotation-vibration coupling, back scattering and recrossing which all may contribute to the final state distribution. Under the present experimental conditions, the orbital angular momentum of complex-forming collisions is computed to be $L \lesssim 140 \hbar$ which is available for conversion into rotational motion of the reaction complex. Coriolis forces may lead to a coupling of the complex rotation to its internal motion, providing another mechanism for the transfer of angular momentum to the fragments. Furthermore, the trajectories shown in Fig. 10 suggest that a wide range of scenarios is possible. They include direct mechanisms, illustrated by the blue trajectory, and can range to bound states with almost full redistribution (randomization, i.e. IVR) of the internal energy as shown for the orange trace. This is reminiscent of the situation recently encountered in the vibrationally induced photodissociation of sulfuric acid where all regimes from prompt reaction to complete IVR were found, depending on the amount of internal energy made available to the molecule 50]. 


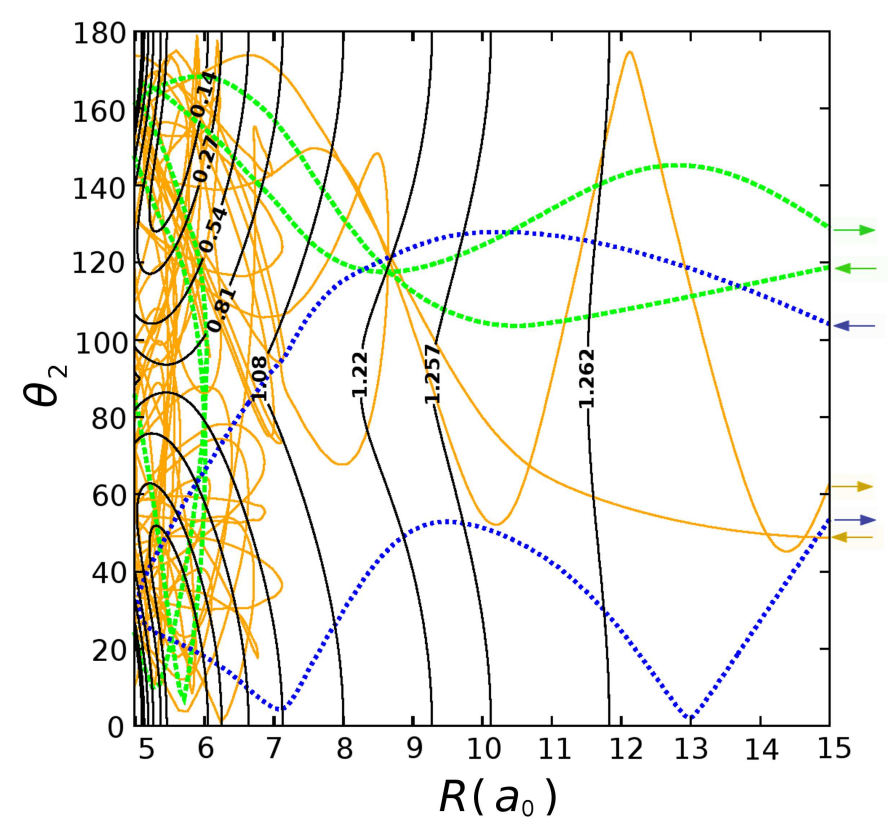

Figure 10: Projection of the PES onto the $R$ and $\theta_{2}$ coordinate (see Figure 6) with $r_{1}=r_{2}=2.08 a_{0}, \theta_{1}=0$, and $\varphi=0$. Contour lines are drawn between 0 and $1.262 \mathrm{eV}$. Three typical trajectories leading to rotational excitation of the products are reported. The arrows indicate the direction of motion (incoming and outgoing). The complex lifetimes amount to $87 \mathrm{fs}$ (blue), $341 \mathrm{fs}$ (green) and 6604 fs (orange).

\section{Summary and conclusions}

In the present study we have demonstrated for the first time ion-molecule reaction studies with state-selected Coulomb-crystallized molecular ions using the symmetric CT reaction $\mathrm{N}_{2}^{+}+\mathrm{N}_{2}$ as an example. By rotational state selection of the reactant ions and their localization in space by sympathetic cooling in an ion trap, their internal and translational motions were completely controlled. By simultaneously cooling the neutral co-reactants to the lowest rotational states and collimating their kinetic-energy distribution in a supersonic molecular beam, ion-molecule reactions were performed with an unprecedented degree of control over both the collision energy and internal states of the reaction partners. The analysis of the product-ion rotational state distribution yielded for the first time information on T-R energy transfer occurring during CT. The experimental results were analyzed and interpreted by making contact with quasi-classical trajectory simulations on a full-dimensional PES. The simulations reproduced the experimental findings and yielded important insights at an atomistic level into the mechanisms underlying T-R energy transfer.

\section{References}

[1] K. Liu, J. Chem. Phys. 125 (2006) 132307.

[2] A. Teslja, J. J. Valentini, J. Chem. Phys. 125 (2006) 132304.

[3] X. Yang, Phys. Chem. Chem. Phys. 13 (2011) 8112.
[4] L. D. Carr, D. DeMille, R. V. Krems, J. Ye, New J. Phys. 11 (2009) 055049.

[5] M. Schnell, G. Meijer, Angew. Chem. Int. Ed. 48 (2009) 6010.

[6] S. Willitsch, Int. Rev. Phys. Chem. 31 (2012) DOI:10.1080/0144235X.2012.667221.

[7] J. J. Gilijamse, S. Hoekstra, S. Y. T. van de Meerakker, G. C. Groenenboom, G. Meijer, Science 313 (2006) 1617.

[8] S. Willitsch, M. T. Bell, A. D. Gingell, S. R. Procter, T. P. Softley, Phys. Rev. Lett. 100 (2008) 043203.

[9] S. Ospelkaus, K.-K. Ni, D. Wang, M. H. G. de Miranda, B. Neyenhuis, G. Quéméner, P. S. Julienne, J. L. Bohn, D. S. Jin, J. Ye, Science 327 (2010) 853.

[10] M. H. G. de Miranda, A. Chotia, B. Neyenhuis, D. Wang, G. Qéméner, S. Ospelkaus, J. L. Bohn, J. Ye, D. S. Jin, Nat. Phys. 7 (2011) 502.

[11] F. H. J. Hall, M. Aymar, N. Bouloufa-Maafa, O. Dulieu, S. Willitsch, Phys. Rev. Lett. 107 (2011) 243202.

[12] K. Mølhave, M. Drewsen, Phys. Rev. A 62 (2000) 011401.

[13] S. Willitsch, M. T. Bell, A. D. Gingell, T. P. Softley, Phys. Chem. Chem. Phys. 10 (2008) 7200.

[14] S. R. Mackenzie, T. P. Softley, J. Chem. Phys. 101 (1994) 10609.

[15] Th. Glenewinkel-Meyer, D. Gerlich, Isr. J. Chem. 37 (1997) 343.

[16] R. J. Green, J. Qian, H.-T. Kim, S. L. Anderson, J. Chem. Phys. 113 (2000) 3002.

[17] R. A. Dressler, Y. Chiu, D. J. Levandier, X. N. Tang, Y. Hou, C. Chang, C. Houchins, H. Xu, C.-Y. Ng, J. Chem. Phys. 125 (2006) 132306.

[18] L. Paetow, F. Unger, W. Beichel, G. Frenking, K.-M. Weitzel, J. Chem. Phys. 132 (2010) 174305.

[19] X. Tong, A. H. Winney, S. Willitsch, Phys. Rev. Lett. 105 (2010) 143001.

[20] X. Tong, D. Wild, S. Willitsch, Phys. Rev. A 83 (2011) 023415.

[21] P. F. Staanum, K. Højbjerre, P. S. Skyt, A. K. Hansen, M. Drewsen, Nat. Phys. 6 (2010) 271.

[22] T. Schneider, B. Roth, H. Duncker, I. Ernsting, S. Schiller, Nat. Phys. 6 (2010) 275.

[23] B. Friedrich, S. L. Howard, A. L. Rockwood, W. E. Trafton, D. Wen-Hu, J. H. Futrell, Int. J. Mass Spectrom. 59 (1984) 203.

[24] P. A. M. van Koppen, M. F. Jarrold, M. T. Bowers, L. M. Bass, K. R. Jennings, J. Chem. Phys. 81 (1984) 288.

[25] M. J. Frost, S. Kato, V. M. Bierbaum, S. R. Leone, J. Chem. Phys. 100 (1994) 6359.

[26] S. Kato, V. M. Bierbaum, S. R. Leone, J. Chem. Phys. 102 (1998) 6659.

[27] K. Sohlberg, Chem. Phys. 246 (1999) 307.

[28] M. J. Frost, C. R. J. Sharpe, Phys. Chem. Chem. Phys. 3 (2001) 4536.

[29] K. B. McAfee, C. R. Szmanda, R. S. Hozack, J. Phys. B: At. Mol. Phys 14 (1981) L243.

[30] K. Sohlberg, J. Futrell, K. Szalewicz, J. Chem. Phys. 94 (1991) 6500.

[31] D. R. Miller, in: G. Socles (Ed.), Atomic and Molecular Beam Methods, volume 1, Oxford University Press, New York, 1988, p. 14.

[32] G. Purvis, R. Bartlett, J. Chem. Phys. 76 (1982) 1910 - 1918.

[33] A. Scheiner, G. Scuseria, J. Rice, T. Lee, H. Schaefer, J. Chem. Phys. 87 (1987) 5361.

[34] T. H. Dunning, J. Chem. Phys. 90 (1989) 1007 - 1024.

[35] M. J. Frisch, G. W. Trucks, H. B. Schlegel, G. E. Scuseria, M. A. Robb, J. R. Cheeseman, J. A. J. Montgomery, T. Vreven, K. N. Kudin, J. C. Burant, J. M. Millam, S. S. Iyengar, J. Tomasi, V. Barone, B. Mennucci, M. Cossi, G. Scalmani, N. Rega, G. A. Petersson, H. Nakatsuji, M. Hada, M. Ehara, K. Toyota, R. Fukuda, J. Hasegawa, M. Ishida, T. Nakajima, Y. Honda, O. Kitao, H. Nakai, M. Klene, X. Li, J. E. Knox, H. P. Hratchian, J. B. Cross, C. Adamo, J. Jaramillo, R. Gomperts, R. E. Stratmann, O. Yazyev, A. J. Austin, R. Cammi, C. Pomelli, J. W. Ochterski, P. Y. Ayala, K. Morokuma, G. A. Voth, P. Salvador, J. J. Dannenberg, V. G. Zakrzewski, S. Dap- 
prich, A. D. Daniels, M. C. Strain, O. Farkas, D. K. Malick, A. D. Rabuck, K. Raghavachari, J. B. Foresman, J. V. Ortiz, Q. Cui, A. G. Baboul, S. Clifford, J. Cioslowski, B. B. Stefanov, G. Liu, A. Liashenko, P. Piskorz, I. Komaromi, R. L. Martin, D. J. Fox, T. Keith, M. A. Al-Laham, C. Y. Peng, A., M. Nanayakkara, P. M. W. Challacombe, B. Gill, Johnson, W. Chen, M. W. Wong, C. Gonzalez, J. A. Pople, Gaussian 03, Revision C.01, Gaussian, Inc., Wallingford CT, U.S.A., 2004.

[36] C. Léonard, P. Rosmus, S. Carter, N. C. Handy, J. Phys. Chem. A 103 (1999) 1846.

[37] K.-M. Weitzel, J. Mähnert, Int. J. Mass. Spectrom. 214 (2002) 175.

[38] B. R. Johnson, J. Chem. Phys. 29 (1985) 1204.

[39] J. A. Nelder, R. Mead, Chem. Phys. 7 (1965) 308.

[40] J. Danielsson, M. Meuwly, J. Chem. Theory. Comput. 4 (2008) 1083.

[41] T. J. Abdulla, J. R. Cash, M. T. Diamantakis, Comput. Math. Appl. 42 (2001) 121.

[42] J. M. Bowman, B. Gazdy, Q. Y. Sun, J. Chem. Phys. 91 (1989) 2859.

[43] W. H. Miller, W. L. Hase, C. L. Darling, J. Chem. Phys. 91 (1989) 2863.

[44] G. H. Peslherbe, W. L. Hase, J. Chem. Phys. 100 (1994) 1179.

[45] R. N. Porter, L. M. Raff, W. H. Miller, J. Chem. Phys. 63 (1975) 2214.

[46] G. Gioumousis, D. P. Stevenson, J. Chem. Phys. 29 (1958) 294.

[47] N. G. Adams, D. Smith, Astrophys. J. Lett. 247 (1981) L123.

[48] T. B. McMahon, P. G. Miasek, J. L. Beauchamp, Int. J. Mass Spectrom. 21 (1976) 63.

[49] L. F. Phillips, J. Chem. Phys. 92 (1990) 6523.

[50] J. Yosa, M. Meuwly, J. Phys. Chem. A 115 (2011) 14350. 